\title{
USING THE SONOGRAPHY IN THE DIAGNOSTICS OF THE ROLLING OF THE ORBIT BOARDS
}

\author{
Akramova Nazima \\ Tashkent State Dental Institute \\ nozima.akramova@gmail.com \\ Orcid ID orcid.org/0000-0002-6155-5793
}

The aim of the study is to assess the possibilities of sonography in the diagnosis of orbital wall fractures

Materials and methods: 21 patients with suspicions of fractures of orbital walls were examined. X-ray films, MSCT scans were taken for all patients and were compared with the ultrasonography findings.

Results: Sonography revealed 18 fractures of 21, which were certified with MSCT. Signs of the fracture not detected in 3 patients, 2 of them were patients with fractures of the lower wall and 1 of the medial wall of the orbit. In 2 cases the fractures of the lateral wall of the orbit were false positive on sonography. Sensitivity of sonography in fractures of the orbital walls was $86 \%$, radiography $81 \%$, respectively. 
Conclusions: Sonography does not replace, but supplement radiography, and allows to get a complete panoramic image of the maxillofacial region. Increasing of the sound conductivity of the bone is the indirect sonography sign of fractures of the lower and lateral walls of the orbit. The causes of sonographic diagnostic errors are fractures without displacement of fragments, minor damage of the cortical layer, small fracture size.

Key words: orbital wall, fractures, sonography

\section{ИСПОЛЬЗОВАНИЕ СОНОГРАФИИ В ДИАГНОСТИКЕ ПЕРЕЛОМОВ СТЕНОК ОРБИТЫ Акрамова Назима Ташкентский государственный стоматологический институт}

Целью исследования является оценка роли и значения сонографии в общем комплексе лучевых методов диагностики переломов стенок орбиты.

Материалы и методы: Проведен анализ данных обследования 21 больных с подозрениями на переломы стенок орбиты. У всех больных выполнены двухмерная серошкальная сонография, рентгенография и многосрезовая компьютерная томография.

Результаты и их обсуждение: При сонографии признаки переломов стенок орбиты выявлены у 18 из 21 больных, с удостоверенными при МСКТ переломами. Признаки перелома не выявлены у 3 больных, в том числе у 2 с переломами нижней стенки и у 1 медиальной стенки орбиты. В 2 случаях сонографически были получены ложноположительные результаты о наличии переломов латеральной стенки орбиты. Чувствительность сонографии при переломах стенок орбиты составила $86 \%$, рентгенографии $81 \%$ соответственно. 
Выводы: сонография не заменяет, а дополняет рентгенографию, при которой возможно получение полного панорамного изображения челюстно-лицевой области. Переломы нижней и наружной стенок орбиты на сонограммах проявляются косвенным признаком усиление звукопроводимости кости. Причинами диагностических ошибок сонографии при травматических повреждениях стенок орбиты являются переломы без смещения отломков с незначительным поврежденнием кортикального слоя, малые размеры перелома.

Ключевые слова: стенки орбиты, перелом, сонография

\section{Актуальность}

Травмы глаза и структур орбиты составляют приблизительно 20,0 \% патологии органа зрения, которые являются основной причиной слепоты и слабовидения лиц детского и трудоспособного возраста. В 50,0 \% случаев повреждения органа зрения приводят к слепоте одного глаза, в 20,0 \% -обоих глаз [4]

В структуре травм органа зрения у взрослых первое место занимают проникающие ранения, составляющие от 67,0 до 84,0 \% [5,7]. У детей около 50,0 \% травм составляют контузии, на долю проникающих ранений приходится не более 5,0% [9]

Сочетанное повреждение нескольких анатомических полиморфизм структур глазного яблока, необходимость выработки оптимальной тактики хирургического лечения требуют применения комплекса методов лучевой диагностики [2]. 
Основным и наиболее доступным лучевым методом исследования больных с повреждениями глаз и структур орбиты остается рентгенологический метод, но в ряде случаев этот метод является малоинформативным [3]

В последние годы метод ультразвуковой диагностики высокого разрешения получил более широкое распространение в офтальмологической практике. Преимущество ультразвукового исследования (УЗИ) связано с его доступностью, высокой информативностью и разрешающей способностью, узким кругом противопоказаний $[6,11]$.

На сегодняшний день увеличилось использование ультразвукового исследования в оценке переломов ЧЛО, за счет быстроты, неинвазивности, относительной дешевизны, портативности метода исследования. Быстрота исследования, отсутствие необходимости специальной подготовки пациентов делают возможным использование сонографии уже в приемном покое, отделениях неотложной хирургии [10].

Не смотря на то, что данный метод не имеет широкое признание, ультразвуковое исследование позволяет выявить различные переломы челюстнолицевой области. Важную диагностическую ценность ультразвуковой метод имеет при наличии кровоизлияний в переднюю камеру и в стекловидное тело, при дислокациях хрусталика, отслойках сетчатки. УЗИ позволяет выявлять инородные тела и судить о глубине их залегания [9]. По данным зарубежных авторов (Незафати и др., 2010) в выявлении переломов ЧЛО чувствительность и специфичность ультразвукового исследования достигает $88.2 \%$ и $100 \%$ соответственно.

Health Sciences

Israel, Yashresh 
Травмы органа зрения характеризуются высокой степенью осложнени и последующей инвалидизацией. В зарубежной и отечественной литератур встречаются лишь отдельные публикации, посвященные вопроса совершенствования лучевой диагностики травм глаза и структур орбит [9-11].

Целью исследования является оценка роли и значения сонографии в общем комплексе лучевых методов диагностики переломов стенок орбиты.

\section{Материалы и методы}

Проведен анализ данных обследования 21 больных в возрасте от 18 до 60 лет, с подозрениями на переломы стенок орбиты. Большинство обследованных больных составили мужчины - 16. У 1 пациента перелом стенок орбиты были изолированные, у 20 множественные и сочетались с повреждениями скулоорбитального комплекса. Среди всех повреждений преобладали переломы латеральной стенки орбиты - у 13 пациентов, дна орбиты - у 16 пациентов. Перелом медиальной стенки встречался у 5 больных, перлом крыши орбиты у 1 пациента.

В рамках использованного протокола у всех больных выполнены двухмерная серошкальная сонография, рентгенография и многосрезовая компьютерная томография. Сонография использовалась для диагностики переломов, а также для мониторинга и контроля репозиции костных отломков интраоперационно после репозиции. Исследования проводили на аппарате SLE-501 (Литва) с линейным датчиком частотой 7,5 МГц в положение пациента лежа на спине, полипозиционно с получением продольных и поперечных срезов. Рентгенография челюстно-лицевой области выполнялась в полуаксиальной проекции. 
Многосрезовая компьютерная томография (MCKT) выполнена в аксиальной проекции с последующей трехмерной реконструкцией на аппарате "Somatom Emotion 6" (Siemens, Германия).

При сонографии оценивались следующие анатомические структуры: кожа, подкожная жировая клетчатка, кортикальный слой костей - латеральной, медиальной стенок и нижнего контура орбиты. Одновременно проводилось исследование здоровой стороны для сопоставления выявленных патологических изменений. Эти структуры оценивались также при МСКТ, кроме того компьютерная томография позволяла дополнительно оценить состояние глубоко расположенных костных и мягкотканных структур челюстно-лицевой области.

МСКТ также явилась референс-методом оценки диагностической эффективности сонографии и рентгенографии.

Контрольную группу составили 20 здоровых лиц, которым была проведена сонография ЧЛО. Кроме того, контролем служили симметричные соответствующие неповрежденные костные структуры.

Результаты и их обсуждение

При сонографическом исследовании пациентов контрольной группы анатомические структуры челюстно-лицевой области проявлялись следующими особенностями: кожа выглядела как гиперэхогенная линейная структура, подкожно-жировой слой - как гипоэхогенная структура с чередующимися тонкими гиперэхогенными соединительнотканными волокнами. Жевательные мышцы выглядели как гомогенные гипоэхогенные участки, разделенные множественными параллельно идущими гиперэхогенными прослойками соединительной ткани. Наружная поверхность подлежащей кости 
характеризовалась в виде гиперэхогенной линии с полным отсутствием дистальной ультразвуковой проводимости (рис.1).

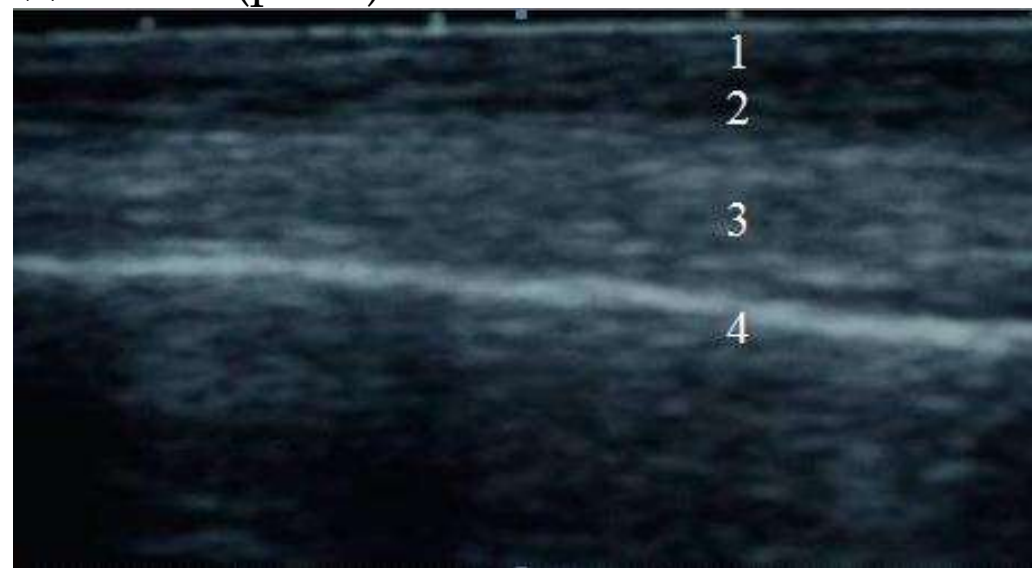

Рис.1. Сонограмма левой скуловой дуги в норме у пациента Ш., 28лет. 1 - кожа; 2 - подкожно-жировая клетчатка; 3 -мышца; 4 - наружный кортикальный слой кости.

Прерывание поверхности кости на сонограммах у здоровых лиц визуализировалось у нижнего края орбиты в месте прохождения нижнеглазничного отверстия. Глазные яблоки проявлялись округлой формы анэхогенными структурами, а медиальная и нижняя стенки орбиты полностью отражали ультразвук с образованием гиперэхогенного контура вокруг дистальных отделов глазных яблок.

Переломы нижней и медиальной стенок орбиты проявлялись косвенным признаком в виде повышения их звукопроводимости. Ушибы мягких тканей приводили к их утолщению, понижению эхогенности с нечеткими границами; подкожные гематомы выглядели как гипо- и анэхогенные участки с четкими границами.

При сонографии признаки переломов стенок орбиты выявлены у 18 из 21 больных, с удостоверенными при МСКТ переломами. При этом переломы стенок орбиты проявлялись только 
косвенным признаком в виде повышения звукопроводимости стенок орбиты (рис. 2а, 2б). Признаки перелома не выявлены у 3 больных, в том числе у 2 с переломами нижней стенки и у 1 медиальной стенки орбиты. У этих больных при компьютерной томографии визуализировалось нарушение целостности кости без смещения отломков.

Ошибка, вероятно, была связана также с большой поверхностью использованного датчика, не позволяющей добиться полного контакта поверхности датчика и кожи в области орбит, что приводило к артефактам на изображении. Подтверждается это исследованиями Friedrich и соавт. [6], которые используя специальный датчик с маленькой поверхностью значительно повысили качество диагностики переломов дна орбиты.

В 2 случаях сонографически были получены ложноположительные результаты о наличии переломов латеральной стенки орбиты, что возможно обусловлено неправильным интерпретированием лобно-скулового шва как перелом.

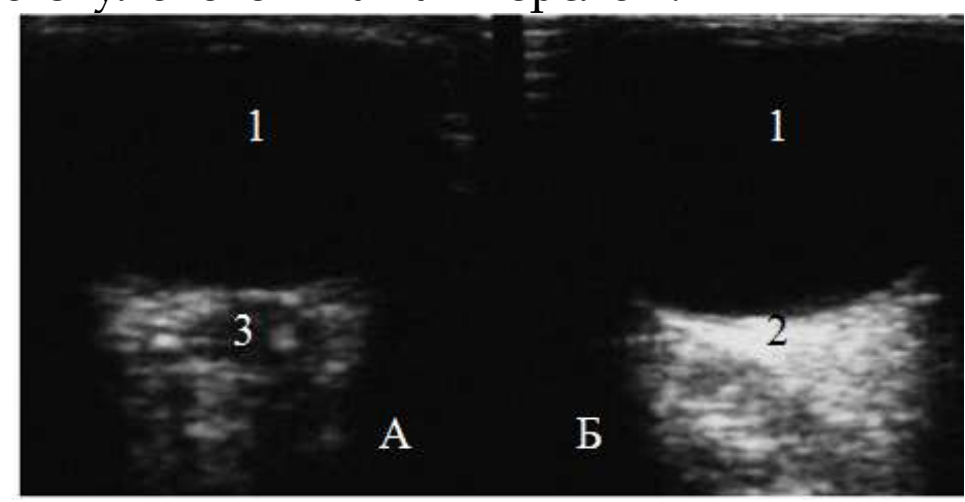

Рис.2а. Сонограммы правой (А) и левой (Б) орбит больного X., 41 год. 1 - глазные яблоки; 2 - нижняя стенка левой орбиты, эхогенность ее сохранена; 3 снижение эхогенности нижней стенки правой орбиты в результате повышение ее звукопроводимости (перелом). 


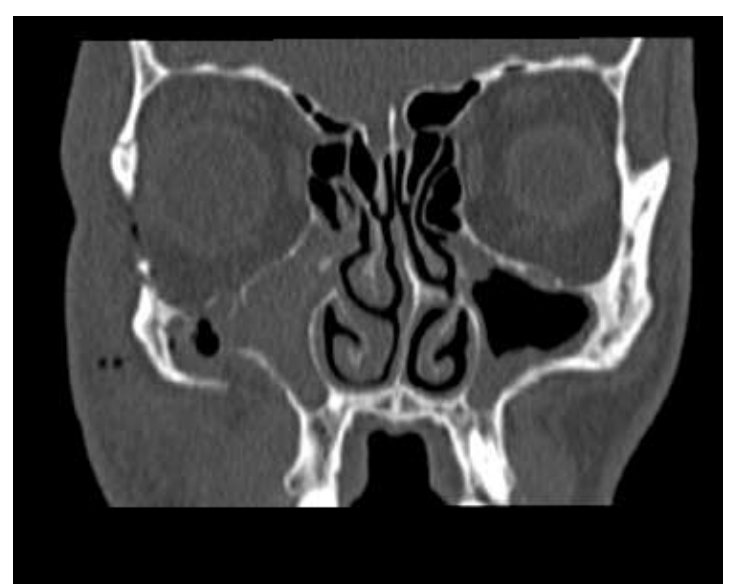

Рис 2б. КТ того же больного, коронарное изображение средней зоны лица. Определяется переломы нижней стенки правой орбиты, скуловой кости и латеральной стенки правой гайморовой пазухи, с пролабированием костных отломков и клетчатки орбиты в сторону пазухи.

Рентгенография не выявила перелом латеральной стенки орбиты в 3 случаях, нижнего контура в 1 случае. Причинами ложноотрицательных результатов были несмещенные переломы, которые затруднили интерпретацию снимка.

При переломах стенок орбит ошибки возникли примерно одинаково часто и при сонографии и при рентгенографии. Поданным других авторов основными причинами являются несмещенные переломы, без повреждения глазничных краев стенок орбит. На это, в частности, указываются и в работах Л.И.Сангаевой (2009), M.Sallam (2010), M.P.Sreeram (2016). По мнению Fredrich и соавторов (2004) качество диагностически переломов стенок орбит улучшается при использовании датчика с маленькой поверхностью, позволяющего добиться полного контакта поверхности датчика и кожи в области орбит [6]. Как показали наши исследования, следует уделять 
внимание такому косвенному признаку, как повышение звукопроводимости стенки орбиты, так как он соответствует ее перелому.
Обладая
ВЫСОКОЙ
специфичностью

рентгенография, к сожалению, не имеет такой же чувствительности, из-за чего некоторые переломы могут быть пропущены. Ошибки в диагностике переломов стенок орбит нередки и при сонографии, в наших исследваниях они имели место в 3 случаяхкак при сонографии так и при рентгенографии. Основной причиной их невыявления являлись неполные переломы. Данное обстоятельство подчеркивает целесообразность применения при травмах указанной локализации компьютерной томографии, не ограничиваясь рентгенографией и сонографией.

Чувствительность сонографии при переломах стенок орбиты составила 86\%, рентгенографии 81\% соответственно. Указанные цифры подчеркивают полезность применения этих взаимодополняющих методов при обследовании больных с травмами ЧЛО. Достоинствами рентгенографии являются высокая специфичность и возможность получения панорамного снимка всем зубочелюстной системы, что важно для распознавания множественных и сочетанных поражений который часто сопровождаются с травмами орбиты. В то же время, сонография оказалась более чувствительной в выявлении переломов стенок орбит.

По данным отечественных авторов [1], сонографию можно использовать интраоперационно и в раннем послеоперационном периоде для определения состояния костных отломков при переломах средней зоны лица при закрытых репозициях[1]. Все это предотвращает лучевую нагрузку на больных. Тем не менее, Friedrich и соавт 
(2003) сообщили что гиперкоррекция скуловой дуги особенно при оскольчатых переломах сонографически плохо визуализируется. Но, при закрытых репозициях интраоперационно строго рекомендуется визуализировать поверхность скуловой дуги [3].

\section{Выводы}

Проведенные исследования показали, что сонография является информативным методом диагностики переломов челюстно-лицевой области, не уступающая рентгенографии. Вместе с тем, сонография не заменяет, а дополняет рентгенографию, при которой возможно получение полного панорамного изображения челюстно-лицевой области.

Переломы нижней и наружной стенок орбиты на сонограммах проявляются косвенным признаком усиление звукопроводимости кости.

Причинами диагностических ошибок сонографии при травматических повреждениях стенок орбиты являются переломы без смещения отломков с незначительным поврежденнием кортикального слоя, малые размеры перелома.

\section{Health Sciences}




\section{References/ Литература}

1. Ходжибеков М.Х., Азимов А.А. Роль сонографии в диагностике и оценке адекватности репозиции переломов костей средней зоны лица // Медицинский журнал Узбекистана, 2011, №6, С. 18-23.

2. Боймурадов Ш.А. Сравнительный анализ результатов лечения больных с сочетанными травмами костей носа и повреждениями головного мозга. Российская оториноларингология. 2009. № 3. С. 31-33.

3. Рабухина Н.А., Буцан С.Б. Использование спиральной КТ для трехмерного компьютерного моделирования при планировании хирургического лечения дефектов и деформаций лицевого скелета // Весник рентгенологии и радиологии, 2009, №: 1., С.1015.

4. Friedrich RE, Heiland M, Bartel-Friedrich S. Potentials of ultrasound in the diagnosis of midfacial fractures. Clin Oral Investig 2003, №7, C. 226-229.

5. Насникова И.Ю., Харлап С.И., Возможности пространственной объемной эхографии в офтальмологической практике // Визуализация в клинике, 2003, № 22-23, С. 4-10.

6. Aburn N.S., Sergott R.C., Color Doppler imaging of the ocular and orbital blood vessels. Curr Opin Ophthalmol. 1993, Vol. 4(6), P. 3-6.

7. Jank S, Emshoff R, Etzelsdorfer M, Strobl H, Nicasi A, Norer B. Ultrasound versus computed tomography in the imaging of orbital floor fractures. J Oral Maxillofac Surg, 2004, Vol. 62, P. 150-154.

8. Nezafati S, Javadrashid R, Rad S, Akrami S. Comparison of ultrasonography with submentovertex films and computed tomo-graphy scan in the diagnosis of zygomatic arch fractures. Dento-maxillofac Radiol, 2010, Vol. 39, P. 11-16. 
9. McCann PJ, Brocklebank LM, Ayoub AF Assessment of zygomatico-orbital complex fractures using ultrasonography. Br J Oral Maxillofac Surg, 2000, P. 525529.

10. Сангаева Л.М. 2009 Лучевая диагностика травм глаза и структур орбиты Автореферат диссертации на соискание ученой степени кандидата медицинских наук Москва, 2009, С.11.

11. Maha Sallam, Ghada Khalifa, Fatma Ibrahim Mohamed Taha. Ultrasonography vs computed tomography in imaging of zygomatic complex fractures Journal of American Science 2010; Vol. 6 (9), P. 524-533

12. Sreeram MP, Rupesh Mandava, Ravindran C, Elengkumaran. Use of ultrasound as a screening tool in the maxillofacial fractures. International Medical Journal, 2016, Vol. 3, 6, P.573-577 